\title{
Numerical Solution of the Time-Depending Flow of Immiscible Fluids with Fuzzy Boundary Conditions
}

\author{
Rajesh Kumar Chandrawat \\ Department of Mathematics, \\ Lovely Professional University, Jalandhar, Punjab, India. \\ Correspondence author: rajesh.16786@lpu.co.in \\ Varun Joshi \\ Department of Mathematics, \\ Lovely Professional University, Jalandhar, Punjab, India. \\ E-mail: varunjoshi20@yahoo.com
}

(Received on February 10, 2021; Accepted on August 2, 2021)

\begin{abstract}
Fluid flow modeling using fuzzy boundary conditions is one of the viable areas in biofluid mechanics, drug suspension in pharmacology, as well as in the cytology and electrohydrodynamic analysis of cerebrospinal fluid data. In this article, a fuzzy solution for the two immiscible fluid flow problems is developed, which is motivated by biomechanical flow engineering. Two immiscible fluids, namely micropolar and Newtonian fluid, are considered with fuzzy boundary conditions in the horizontal channel. The flow is considered unsteady and carried out by applying a constant pressure gradient in the $X$-direction of the channel. The coupled partial differential equations are modeled for fuzzy profiles of velocity and micro-rotation vectors then the numerical results are obtained by the modified cubic B - spline differential quadrature method. The evolution of membership grades for velocity and microrotation profiles has been depicted with the fuzzy boundaries at the channel wall. It is observed that Micropolar fluid has a higher velocity change than Newtonian fluid, and both profiles indicate a declining nature toward the interface.
\end{abstract}

Keywords- Fuzzy boundary conditions, Immiscible fluids, Unsteady flow, Differential quadrature method.

\section{Introduction}

Fluid flow problems with the fuzzy inference control system have a variety of applications in transpiration convection of rocket engines and gas turbines, heat relaxation conditions in buildings, modeling of cross-flow desalination processes, and physiological fluid flow transportation in the blood vessels. To gain the best approximation of complex scientific problems, Chetia and Hazarika (2000) investigated the impact of fuzziness on dynamical similarity and Reynold's number. Many authors have examined uncertainties in the Couette, Magnetohydrodynamics fluid flow problems with fuzzy conditions (Barhoi et al., 2018; Borah et al., 2020). Rajeev (2008) worked at three spatial dimensional fuzzy fluid dynamics by constraining flow velocity. He suggested a spin consistent small range cut-off in the formulation of an ideal fluid flow. Chen et al. (2018) proposed a new approach for solving two-dimensional Navier-Stokes equations with a fuzzy technique.

Many physiologically useful fluids do not follow the Newtonian law of viscosity and are known as non-Newton fluids. Micropolar fluid is one of the non-Newtonian fluids that has drawn the interest of many researchers across the globe. Micropolar fluids comprise animal blood, lubricating solutions, complicated physiological structures, and some copolymer mixtures. Micro polarity was defined and discussed by Eringen (1968). Eringen micropolar fluid comprises tiny-sized microelements that display angular motion as a microrotation vector and have the spine effect. 
Peddieson (1972) obtained empirical results, for balanced shear segment flow from a micropolar fluid. Apparao et al. (2013) discovered that a micropolar fluid seems to be a more prominent strain passage through a channel than a Newtonian fluid. The microstructure's impact on the blood's physicochemical characteristics was detailed by Kang and Eringen (1976). Devakar and Iyengar (2013) explored the time-dependent development of micropolar fluid in two channels. In a particular corridor with a limited real canal, Mekheimer and El Kot (2008) explored blood supplies utilizing the micro-structured liquid. Wang et al. (2016) inspected the movement of micropolar liquids into a smaller scale parallel framework. Some current examinations and overviews on micropolar liquid forms can be seen in (Abdel-Wahed, 2017; Akbar et al., 2018; Farooq et al., 2019; Ziabakhsh et al., 2009).

Major advancements in hydrology, materials processing, and contemporary oil production gear have all been linked to the immiscible viscous fluid. Borrelli et al. (2017) considered two immiscible Newtonian liquids in the duct and examined the magneto-convection impacts on streamline behavior. The thermal, entropy generation, and hydrodynamic analysis of two immiscible fluids through the porous wall are done by Srinivas et al. (2015), Srinivas and Murthy (2016a), Srinivas and Murthy (2016b). Umavathi et al. (2009) proposed a solution of two immiscible liquid flows in a composite permeable media for the fully formed laminar flow between parallel plates. In addition, there are some brief findings in (Bird, 2002; Singh, 2005; Singh et al., 2004; Umavathi et al., 2005; Umavathi et al., 2008) about the behavior of immiscible laminar fluids flowing between parallel plates.

The time constraint in the flow processing is one of the important aspects thus, the consideration of the time-dependent stream of immiscible fluids is perfect for their biomechanical and hydrological applications (Ali et al., 2019; Chamkha et al., 2020; Hamid et al., 2019; Jiang et al., 2018). Arora and Bhatia (2020) applied the radial basis function in the time-dependent differential equation. There are several numerical techniques such as the Crank-Nicolson scheme, explored by Shuaib et al. (2020), and the finite element method (FEM) by Ali et al. (2019) for dealing with the unstable time-dependent flow. Devakar and Raje (2018) numerically investigated the timedependent flow of some immiscible fluid through a duct by the Crank-Nicolson scheme. The differential quadrature (DQ) approach proposed by Bellman et al. (1972) is one of the suitable numerical techniques to simulates the linear and nonlinear coupled partial differential equations. Quan and Chang (1989a, 1989b) expanded the DQM by modifying the basis function. Different other test functions such as Lagrange addition polynomials, sink, and spline, cubic, and modifiedcubic B-spline are utilized in (Arora et al., 2019; Civan, 1993; Jiwari and Alshomrani, 2017; Mittal and Jain, 2012; Shan et al., 2008; Shu et al., 2007; Shu and Richards, 1992) for the calculation of weighting coefficients in DQM. Vaid and Arora (2019) used the quintic spline basis function to solve the delay differential equation. The differential quadrature method provides a good agreement for approximating the derivatives and solved the unsteady flow problem (Katta et al., 2020b; Ramesh and Joshi, 2019).

The physiological flow phenomena become uncertain with various imprecise characteristics and conditions (Chaube et al., 2010; Katta et al., 2020a; Tripathi et al., 2021). It is difficult to provide accurate predictions with crisp variables and conditions. The simulation with fuzzy variables and boundary conditions can provide a better degree for the decision-making process in biomedical and microfluidics processes. Given the importance of micropolar liquids, no effort was made to investigate the unstable consistency of fuzzy boundary conditions for two immiscible micropolar and Newtonian fluids. In this paper, we considered the time-dependent flow of two immiscible 
micropolar blood and Newtonian fluids with fuzzy boundary conditions in the horizontal channel. The fuzzy solution is obtained numerically by solving coupled partial differential equations using the modified cubic B-spline differential quadrature method.

\subsection{Nomenclature}

The following Table 1 describes the different notations/symbols used in the paper.

Table 1. The nomenclature used for various symbols and notations.

\begin{tabular}{|c|l|c|l|}
\hline$\rho$ & Density co-efficient & $u$ & Velocity of fluid \\
\hline$\gamma_{1}, \mu, \delta$ & Viscosity coefficients & $\omega$ & Microrotation \\
\hline$f$ & Body force per unit mass & $\xi$ & Body couple per unit mass \\
\hline$\lambda_{1}, \lambda_{2}, \lambda_{3}$ & Gyro-viscosity coefficients & $\tau$ & Gyration co-efficient \\
\hline$p$ & Fluid pressure at any point & $u_{f_{1}}$ & The velocity of micropolar fluid \\
\hline$\rho_{1}, \mu_{1}$ & Density, the viscosity of the micropolar fluid & $u_{f_{2}}$ & The velocity of the Newtonian fluid \\
\hline$\rho_{2}, \mu_{2}$ & Density, the viscosity of the Newtonian fluid & $\omega_{f_{1}}$ & Microrotation of micropolar fluid \\
\hline$\delta_{1}$ & Vortex viscosity of the micropolar fluid & $\alpha_{c}$ & An alpha cut of fuzzy set \\
\hline$\eta_{1}$ & Micropolar parameter & $R e$ & Reynolds number \\
\hline$r_{1}$ & Ratio of viscosity & $r_{2}$ & Ratio of density \\
\hline$W_{i, j}^{(1)}$ & Weighting coefficient of $1^{\text {st }}$ order derivative & $W_{i, j}^{(2)}$ & Weighting coefficient of $2^{\text {nd }}$ order derivative \\
\hline
\end{tabular}

\section{Basic Equations}

The micropolar fluid flow governing equations are provided by Eringen (1968).

$$
\rho_{t}+\nabla \cdot(\rho u)=0
$$

$\rho \cdot \frac{\partial u}{\partial t}=\rho f-\nabla p+\delta \nabla \times \omega-(\mu+\delta) \nabla \times \nabla \times u+\left(\gamma_{1}+2 \mu+\delta\right) \nabla(\nabla \cdot u)$

$$
\rho . \tau \frac{\partial \omega}{\partial t}=\rho \xi-2 \delta \omega+\delta \nabla \times u-\lambda_{1} \nabla \times \nabla \times \omega+\left(\lambda_{1}+\lambda_{2}+\lambda_{3}\right) \nabla(\nabla . \omega)
$$

Here $\rho$ is the density co-efficient, $u$ is the velocity of fluid, $\gamma_{1}, \mu$ and $\delta$ are viscosity coefficients, $\omega$ is the microrotation, $f$ is the body force per unit mass, $\xi$ is the body couple per unit mass, $\tau$ is the gyration co-efficient, $\lambda_{1}, \lambda_{2}$ and $\lambda_{3}$ are gyro-viscosity coefficients, $p$ is the fluid pressure at any point. In the absence of micro rotational effect $(\omega=0, \delta \rightarrow 0)$, equation (2) can be reduced to the following Newtonian fluid flow equation.

$$
\rho \cdot \frac{\partial u}{\partial t}=\rho f-\nabla p-\mu \nabla \times \nabla \times u+\left(\gamma_{1}+2 \mu\right) \nabla(\nabla . u)
$$

\section{Mathematical Formulation}

Consider the unsteady, laminar, and unidirectional flow of two immiscible - micropolar and Newtonian fluids - undergoing shearing motions. The following assumptions are being made for the momentum of the flow:

- Both fluids are viscous and move between two horizontal parallel non-porous plates.

- Both plates are believed to be electrically non-conductive. The plates are located in the $X-Z$ plane as depicted in Figure 1. 


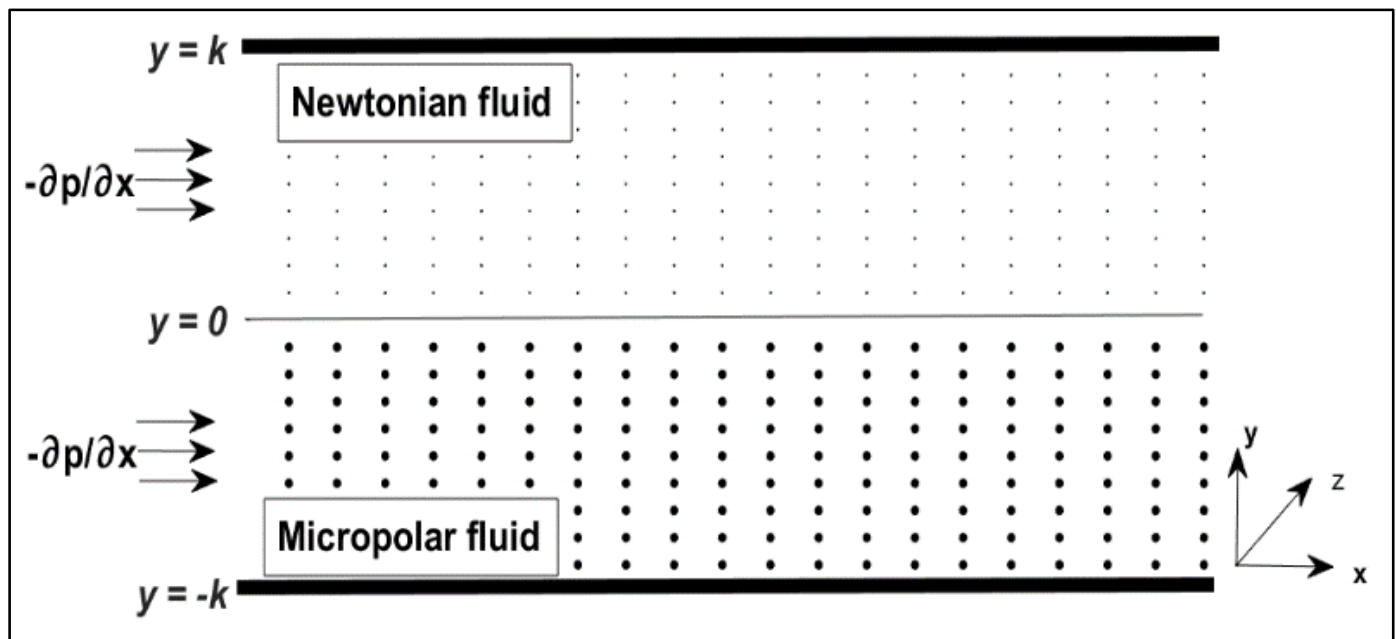

Figure 1. The geometrical configuration of the mean flow of two immiscible fluids.

- Both plates are stable while the flow get momentum by the uniform pressure gradient applied from $x$-direction and maximum flow velocity is $U_{0}$.

- Let $\rho_{1}, \mu_{1}$ be the density and viscosity of micropolar fluid which lies in $(-k \leq y \leq 0)$ and possesses a linear fluid velocity $u_{f_{1}}$, microrotation (angular velocity) $\omega_{f_{1}}$, vortex viscosity $\delta_{1}$, gyro-viscosity $\lambda_{1}$, and gyration parameter $\tau$.

- The Newtonian fluid has a density $\rho_{2}$ and viscosity $\mu_{2}$, fluid velocity $u_{f_{2}}$ and occupies the region $(0 \leq y \leq k)$.

- $\quad$ Both fluids are incompressible so $\nabla . u_{f_{1}}=0, \nabla . u_{f_{2}}=0$.

- The evolution of velocity and micro-rotation profiles is analyzed by using uncertain boundary conditions. Let $x$ be the fuzzy variable then the fuzzy boundary condition for both velocity and micro- rotation profiles is $\tau_{b, d_{c}, i_{c}}(x)$ defined as:

Let $X$ be the universal space of fuzzy variable and $a, b$ and $c \in X$ such that $a<b<c$ then a triangular fuzzy number $\tau_{b, d_{c}, i_{c}}$ with membership grade $\tau_{b, d_{c}, i_{c}}(x)$ is defined as:

$\tau_{b, d_{c}, i_{c}}(x)=\left\{\begin{array}{cc}0 & x<a \\ \frac{x-a}{b-a} & a<x<b \\ \frac{c-x}{c-b} & b<x<c \\ 0 & x>c\end{array}\right\}$ where $d_{c}=b-a, i_{c}=c-b$

Figure 2 represents the membership grade of $\tau_{b, d_{c}, i_{c}}$. 


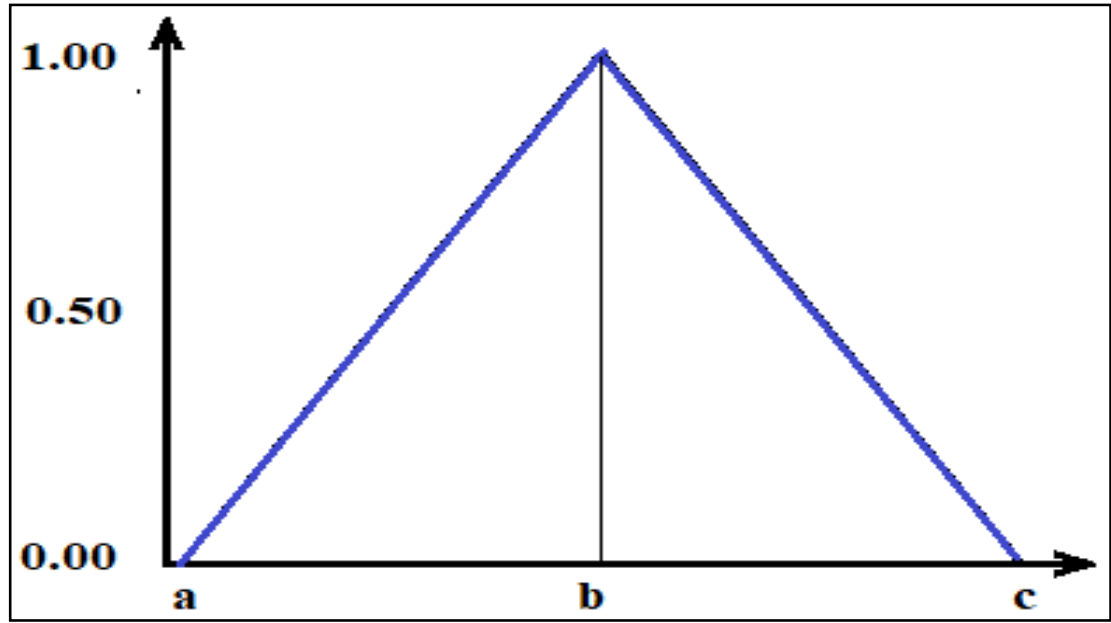

Figure 2. Triangular fuzzy number $\boldsymbol{\tau}_{\boldsymbol{b}, \boldsymbol{d}_{c}, \boldsymbol{i}_{\boldsymbol{c}}}$ with its membership grade.

- The flow is unidirectional so linear and angular velocities for both region fluids are assumed as:

$$
u_{f_{1}}=\left(u_{f_{1}}(y, t), 0,0\right), u_{f_{2}}=\left(u_{f_{2}}(y, t), 0,0\right) \text {, and } \omega_{f}=\left(0,0, \omega_{f}(y, t)\right) \text {. }
$$

Using the aforementioned assumptions, the two-fluid governing equations (2), (3), and (4) can then be simplified as:

Region-I for Micropolar fluid $(-k \leq y \leq 0)$ :

$\rho_{1} \frac{\partial u_{f_{1}}}{\partial t}=-\frac{\partial p}{\partial x}+\delta_{1} \frac{\partial \omega_{f_{1}}}{\partial y}+\left(\mu_{1}+\delta_{1}\right) \frac{\partial^{2} u_{f_{1}}}{\partial y^{2}}$

$\rho_{1} \tau \frac{\partial \omega_{f_{1}}}{\partial \mathrm{t}}=\lambda_{1} \frac{\partial^{2} \omega_{f_{1}}}{\partial y^{2}}-\delta_{1}\left(2 \omega_{f_{1}}+\frac{\partial u_{f_{1}}}{\partial y}\right)$

Region-II for Newtonian fluid $(0 \leq \mathrm{y} \leq \mathrm{k})$ :

$\rho_{2} \frac{\partial u_{f_{2}}}{\partial t}=-\frac{\partial p}{\partial x}+\mu_{2} \frac{\partial^{2} u_{f_{2}}}{\partial y^{2}}$

The fuzzy conditions are considered at the boundaries with the alpha cut $\alpha_{c} \rightarrow[0,1]$ of fuzzy set. These conditions can be written as follows in numerical terms.

Initial conditions: At $\mathrm{t} \leq 0$,

$$
\left.\begin{array}{cc}
u_{f_{1}}(y, t)=0 & \text { for }-k \leq y \leq 0 \\
\omega_{f_{1}}(y, t)=0 & \text { for }-k \leq y \leq 0 \\
u_{f_{2}}(y, t)=0 & \text { for } 0 \leq y \leq k
\end{array}\right\}
$$

Fuzzy boundary and interfacial conditions: At $t>0$, 


$$
\begin{aligned}
& u_{f_{1}}(-k, t)=\left(\underline{u_{f_{1}}(-k, x)}, u_{f_{1}}(-k, x), \overline{u_{f_{1}}(-k, x)}\right)=\left(\alpha_{c}, 1,2-\alpha_{c}\right) \\
& u_{f_{2}}(k, t)=\left(u_{f_{2}}(k, x), u_{f_{2}}(k, x), \overline{u_{f_{2}}(k, x)}\right)=\left(\alpha_{c}, 1,2-\alpha_{c}\right) \\
& \left.\omega_{f_{1}}(-k, t)=\left(\underline{\omega_{f_{1}}(-k, x)}, \omega_{f_{1}}(-k, x), \overline{\omega_{f_{1}}(-k, x)}\right)=\left(\alpha_{c}, 1,2-\alpha_{c}\right)\right\} \\
& u_{f_{1}}(0, t)=u_{f_{2}}(0, t) \\
& \omega_{f_{1}}(0, t)=-\frac{1}{2} \frac{\partial u_{f_{1}}}{\partial t} \\
& \mu_{2} \frac{\partial u_{f_{2}}}{\partial y}=\left(\mu_{1}+\delta_{1}\right) \frac{\partial u_{f_{1}}}{\partial y}+\omega_{f_{1}} \delta_{1} \text { at } y=0
\end{aligned}
$$

Introducing the non-dimensional parameters:

$$
\begin{gathered}
\bar{x}=\frac{x}{k}, \bar{y}=\frac{y}{k}, \overline{u_{f_{1}}}=\frac{u_{f_{1}}}{U_{0}}, \overline{u_{f_{2}}}=\frac{u_{f_{2}}}{U_{0}}, \overline{\omega_{f_{1}}}=\frac{\omega_{f_{1}}}{U_{0}} k, \bar{p}=\frac{p}{\rho_{1} U_{0}{ }^{2}}, \\
\bar{t}=\frac{t U_{0}}{k}, \lambda_{1}=\left(\mu_{1}+\frac{\delta_{1}}{2}\right) \tau, \text { with } \tau=k^{2}, \text { Micropolar parameter } \eta_{1}=\frac{\delta_{1}}{\mu_{1}}
\end{gathered}
$$

Reynolds number $\operatorname{Re}=\frac{\rho_{1} U_{0}}{\mu_{1}}$, the ratio of viscosity $r_{1}=\frac{\mu_{2}}{\mu_{1}}$, and density $r_{2}=\frac{\rho_{2}}{\rho_{1}}$ )

The constant pressure $G e(t)-\nabla \rho=-\partial \rho / \partial x$ with $t>0$ is considered to get numerical results.

After dropping the bars and introducing the above non-dimensional parameters, appropriate initial, interfacial, and fuzzy boundary conditions, the equations (6)-(8) can be simplified as:

Region-I ( $-k \leq y \leq 0)$ (Micropolar fluid region):

$\frac{\partial u_{f_{1}}}{\partial t}=G e(t)+\frac{n_{1}}{R e} \frac{\partial \omega_{f_{1}}}{\partial y}+\frac{\left(n_{1}+1\right)}{R e} \frac{\partial^{2} u_{f_{1}}}{\partial y^{2}}$

$\frac{\partial \omega_{f_{1}}}{\partial \mathrm{t}}=\frac{\left(\frac{n_{1}}{2}+1\right)}{R e} \frac{\partial^{2} \omega_{f_{1}}}{\partial y^{2}}-\frac{n_{1}\left(2 \omega_{f_{1}}+\frac{\partial u_{f_{1}}}{\partial y}\right)}{R e}$

Region-II for Newtonian fluid $(0 \leq y \leq k)$ :

$\frac{\partial u_{f_{2}}}{\partial t}=\frac{G e(t)}{r_{2} R e}+\frac{r_{1}}{r_{2} R e} \frac{\partial^{2} u_{f_{2}}}{\partial \mathrm{y}^{2}}$

Equation (9)-(10) are considered as initial and fuzzy boundary conditions with $k=1$.

\section{Numerical Solution}

To analyze the fuzzy solution for velocity and microrotation profile with fuzzy boundaries, the microrotation vector, and micropolar fluid velocity is calculated in the domain $(-1 \leq y \leq 0)$ using the velocity vector of micropolar fluid, and then the velocity vector of Newtonian fluid is obtained in $(0 \leq y \leq 1)$. We employ unified discretization of the domain $[a, b]$ in spline-based differential quadrature method with phase length $h: a=y_{1}<y_{2}<\cdots<y_{n-1}<y_{n}=b$, such that $y_{i+1}-$ $y_{i}=h$ on the real axis. The $1^{s t}$ and $2^{\text {nd }}$ order derivatives of $u_{f_{1}}, \omega_{f_{1}}$, and $u_{f_{2}}$ are obtained concerning y as: For $i=1,2,3, \ldots, n$ and $j=1,2, \ldots, N$. 
$\frac{\partial \omega_{f_{1}}}{\partial y}=\sum_{j=1}^{N} W_{i, j}^{(1)} \omega_{f_{1}}\left(y_{j}, t\right)$

$\frac{\partial^{2} \omega_{f_{1}}}{\partial \mathrm{y}^{2}}=\sum_{j=1}^{N} W_{i, j}^{(2)} \omega_{f_{1}}\left(y_{j}, t\right)$

$\frac{\partial u_{f_{1}}}{\partial \mathrm{y}}=\sum_{j=1}^{N} W_{i, j}^{(1)} u_{f_{1}}\left(y_{j}, t\right)$

$\frac{\partial^{2} u_{f_{1}}}{\partial \mathrm{y}^{2}}=\sum_{j=1}^{N} W_{i, j}^{(2)} u_{f_{1}}\left(y_{j}, t\right)$

$\frac{\partial u_{f_{2}}}{\partial \mathrm{y}}=\sum_{j=1}^{N} W_{i, j}^{(1)} u_{f_{2}}\left(y_{j}, t\right)$

$\frac{\partial^{2} u_{f_{2}}}{\partial \mathrm{y}^{2}}=\sum_{j=1}^{N} W_{i, j}^{(2)} u_{f_{2}}\left(y_{j}, t\right)$

Here $W_{i, j}^{(1)} W_{i, j}^{(2)}$ are the weighting coefficients of $1^{s t}$ and $2^{\text {nd }}$ order derivatives (Katta et al., 2020b; Ramesh and Joshi, 2019) for $\mathrm{n}$ discrete knots $\left(y_{1}, y_{2}, \ldots y_{n}\right)$ derived from the updated B-spline functions. The cubic B-spline at knots is defined as Katta et al. (2020b).

$\varphi_{j}(y)=\frac{1}{h^{3}}\left\{\begin{array}{cc}\left(y-y_{j-2}\right)^{3}, & y \in\left[y_{j-2}, y_{j-1}\right) \\ \left(y-y_{j-2}\right)^{3}-4\left(y-y_{j-1}\right)^{3}, & y \epsilon\left[y_{j-1}, y_{j}\right) \\ \left(y_{j+2}-y\right)^{3}-4\left(y_{j+1}-y\right)^{3}, & y \epsilon\left[y_{j}, y_{j+1}\right) \\ \left(y_{j+2}-y\right)^{3}, & y \epsilon\left[y_{j+1}, y_{j+2}\right) \\ 0, & \text { otherwise. }\end{array}\right.$

where $\left\{\varphi_{0}(y), \varphi_{1}(y), \varphi_{2}(y) \ldots, \varphi_{n+1}(y)\right\}$ are the basis function over the region $[a, b]$. The basis function is updated as follows to get a system of equations that could be expressed by a diagonaldominated matrix, Katta et al. (2020b).

$$
\left.\begin{array}{c}
\psi_{1}(y)=\varphi_{1}(y)+2 \varphi_{0}(y) \\
\psi_{2}(y)=\varphi_{2}(y)-\varphi_{0}(y) \\
\psi_{j}(y)=\varphi_{j}, \text { for } j=3, \ldots . N-2 \\
\psi_{N-1}(y)=\varphi_{N-1}(y)-\varphi_{N+1}(y) \\
\psi_{N}(y)=\varphi_{N}(y)+2 \varphi_{N+1}(y)
\end{array}\right\}
$$

The derivative of the basic functions are as follows:

$$
\varphi_{j}{ }^{\prime}(y)=\frac{1}{h^{3}}\left\{\begin{array}{cc}
3\left(y-y_{j-2}\right)^{2}, & y \in\left[y_{j-2}, y_{j-1}\right) \\
3\left(y-y_{j-2}\right)^{2}-12\left(y-y_{j-1}\right)^{2}, & y \in\left[y_{j-1}, y_{j}\right) \\
-3\left(y_{j+2}-y\right)^{2}+12\left(y_{j+1}-y\right)^{2}, & y \in\left[y_{j}, y_{j+1}\right) \\
-3\left(y_{j+2}-y\right)^{2}, & y \in\left[y_{j+1}, y_{j+2}\right) \\
0, & \text { otherwise. }
\end{array}\right.
$$




$$
\left.\begin{array}{c}
\psi^{\prime}{ }_{1}(y)=\varphi^{\prime}{ }_{1}(y)+2 \varphi^{\prime}{ }_{0}(y) \\
\psi^{\prime}{ }_{2}(y)=\varphi^{\prime}{ }_{2}(y)-\varphi^{\prime}{ }_{0}(y) \\
\psi^{\prime}{ }_{j}(y)=\varphi^{\prime}{ }_{j}, \text { for } j=3, \ldots . N-2 \\
\psi^{\prime}{ }_{N-1}(y)=\varphi^{\prime}{ }_{N-1}(y)-\varphi^{\prime}{ }_{N+1}(y) \\
\psi^{\prime}{ }_{N}(y)=\varphi^{\prime}{ }_{N}(y)+2 \varphi^{\prime}{ }_{N+1}(y)
\end{array}\right\}
$$

The estimate of the first-order derivative is:

$$
\begin{array}{ll}
\psi_{k}^{\prime}\left(y_{i}\right)=\sum_{j=1}^{N} W_{i, j}^{(1)} \varphi_{k}\left(y_{j}\right) \quad & \text { for } i=1,2, \ldots, N \\
k=1,2, \ldots, N
\end{array}
$$

Then using $W_{i, j}^{(1)}$ for $i=1,2, \ldots, N$ in equation (18) the following tri-diagonal system of equation is established as:

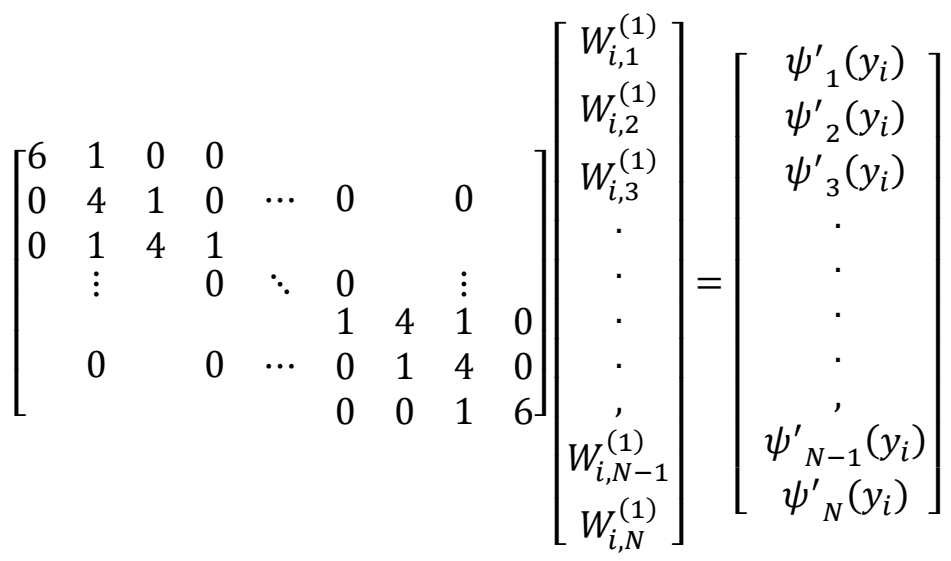

Solving the above system the weighting coefficients $\left\{W_{1,1}^{(1)}, W_{1,2}^{(1)} \ldots, W_{i, N}^{(1)}\right\},\left\{W_{2,1}^{(1)}\right.$ $\left., W_{2,2}^{(1)} \ldots, W_{2, N}^{(1)}\right\}, \ldots,\left\{W_{N, 1}^{(1)}, W_{N, 2}^{(1)} \ldots, W_{N, N}^{(1)}\right\}$ of $1^{\text {st }}$ order derivatives of linear and angular velocities are obtained and then the value of $W_{i, j}^{(2)}$ for $i=1,2,3 \ldots N, j=1,2,3 \ldots N$ is calculated as follows: $W_{i, j}^{(2)}=2 W_{i, j}^{(1)}\left(W_{i, j}^{(1)}-\frac{1}{y_{i}-y_{j}}\right)$ for $\left.i \neq j\right\}$ $\left.W_{i, i}^{(2)}=-\sum_{i=1, i \neq j}^{N} W_{i, j}^{(2)} \quad i=j\right\}$

Substituting the estimation of the $1^{\text {st }}$ and $2^{\text {nd }}$ order derivatives by MCB-DQM scheme, equations (12)-(14) can be written as:

Region-I ( $-k \leq y \leq 0)$ (Micropolar fluid region):

$\frac{\partial u_{f_{1}}}{\partial t}=G e(t)+\frac{n_{1}}{R e}\left(\sum_{j=1}^{N} W_{i, j}^{(1)} \omega_{f_{1}}\left(y_{j}, t\right)\right)+\frac{\left(n_{1}+1\right)}{R e}\left(\sum_{j=1}^{N} W_{i, j}^{(2)} u_{f_{1}}\left(y_{j}, t\right)\right)$

$\frac{\partial \omega_{f_{1}}}{\partial \mathrm{t}}=\frac{\left(n_{1}+2\right)}{2 R e}\left(\sum_{j=1}^{N} W_{i, j}^{(2)} \omega_{f_{1}}\left(y_{j}, t\right)\right)-\frac{n_{1}}{R e}\left(2 \omega_{f_{1}}+\sum_{j=1}^{N} W_{i, j}^{(1)} u_{f_{1}}\left(y_{j}, t\right)\right)$

In the region $(-\mathrm{k} \leq \mathrm{y} \leq \mathrm{k})$ :

$\frac{\partial u_{f_{2}}}{\partial t}=\frac{r_{1}}{R e \cdot r_{2}}\left(\sum_{j=1}^{N} W_{i, j}^{(2)} u_{f_{2}}\left(y_{j}, t\right)\right)+\frac{G e(t)}{r_{2 .}}$ 
In every step of the scheme, the equation (9)-(10) are considered as initial and fuzzy boundary conditions with $k=1$.

Thus, the linear and angular velocities $u_{f_{1}}, u_{f_{2}}$, and $\omega_{f_{1}}$ in equations (23)-(24) with fuzzy boundaries (9)-(10) are being reduced in the following system of differential equations in time, that is, represented as: for $i=1,2,3 \ldots, N$.

$U_{t}=R_{t}\left(u_{f_{1}}, \omega_{f_{1}}, u_{f_{2}}\right)$

The system is solved by the following strong stability B-spline RK43 scheme (Katta et al., 2020b; Ramesh and joshi, 2019).

$$
\begin{aligned}
& U_{1}=U_{0}+\frac{\Delta t}{2} * R_{0}\left(u_{f_{1}}, \omega_{f_{1}}, u_{f_{2}}\right) \\
& U_{2}=U_{1}+\frac{\Delta t}{2} * R_{1}\left(u_{f_{1}}, \omega_{f_{1}}, u_{f_{2}}\right) \\
& U_{3}=\frac{2 U_{0}}{3}+\frac{U_{1}}{3}+\frac{\Delta t}{6} * R_{2}\left(u_{f_{1}}, \omega_{f_{1}}, u_{f_{2}}\right) \\
& U_{M}=U_{3}+\frac{\Delta t}{2} * R_{3}\left(u_{f_{1}}, \omega_{f_{1}}, u_{f_{2}}\right)
\end{aligned}
$$

\section{Result and Analysis}

The unstable flow of two immiscible micro polar blood and Newtonian fluid with fuzzy boundary conditions is examined in the horizontal channel based on the time-dependent pressure gradient, and the fuzzy solution for velocity and micro rotation vector profiles has been achieved.

The obtained numerical results are validated by comparing them in the case of, non-micropolar (angular velocity $\omega_{f_{1}}=0$, vortex viscosity $\delta_{1}=0$, which leads to the Micropolar parameter $n_{1}=$ $\frac{\delta_{1}}{\mu_{1}}=0$ ) and single Newtonian fluid in the horizontal channel with the exact solutions.

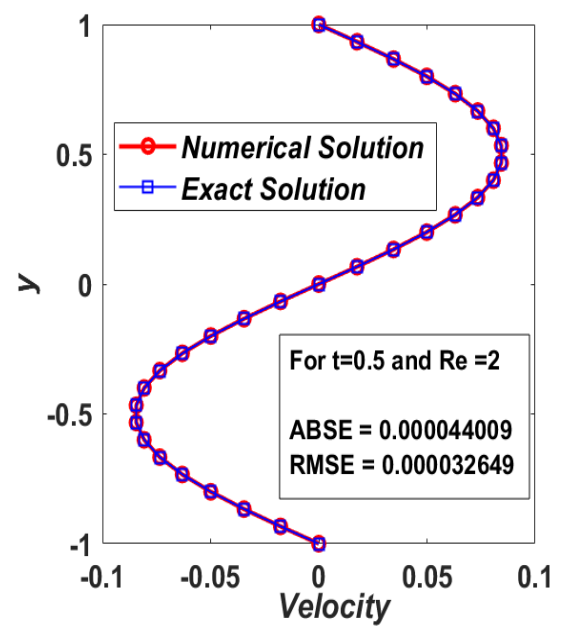

Figure 3. Comparison of present numerical result with an exact solution at $t=0.5$.

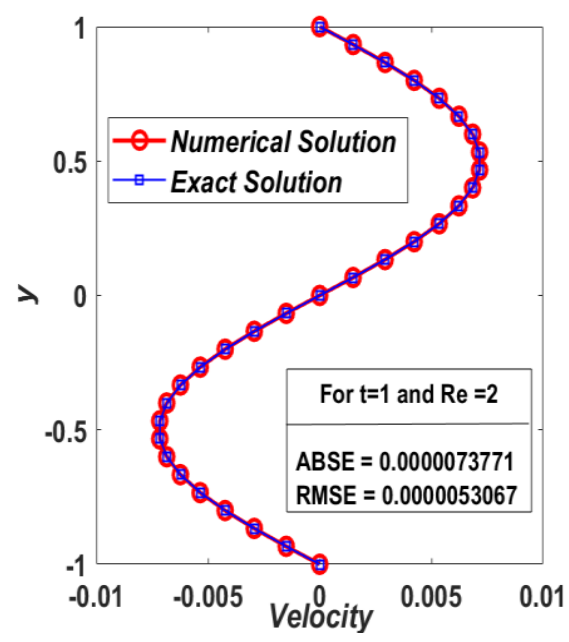

Figure 4. Comparison of present numerical result with an exact solution at $t=1$. 
It is observed from above Figure 3 and Figure 4, that a good agreement is reported between the numerical and the exact solution for the limiting case. The absolute and root mean square errors very less and also declining with time. It is also worth mentioning that the accuracy of the method increases by rising the discretized points.

The fuzzy solution, the evolution of membership grade, and grayscale image of the solution are obtained with the following constant value of the parameters $G e=10, \operatorname{Re}=2, r_{1}=0.5, r_{2}=0.5, n_{1}$ $=0.5, t=0.5$.

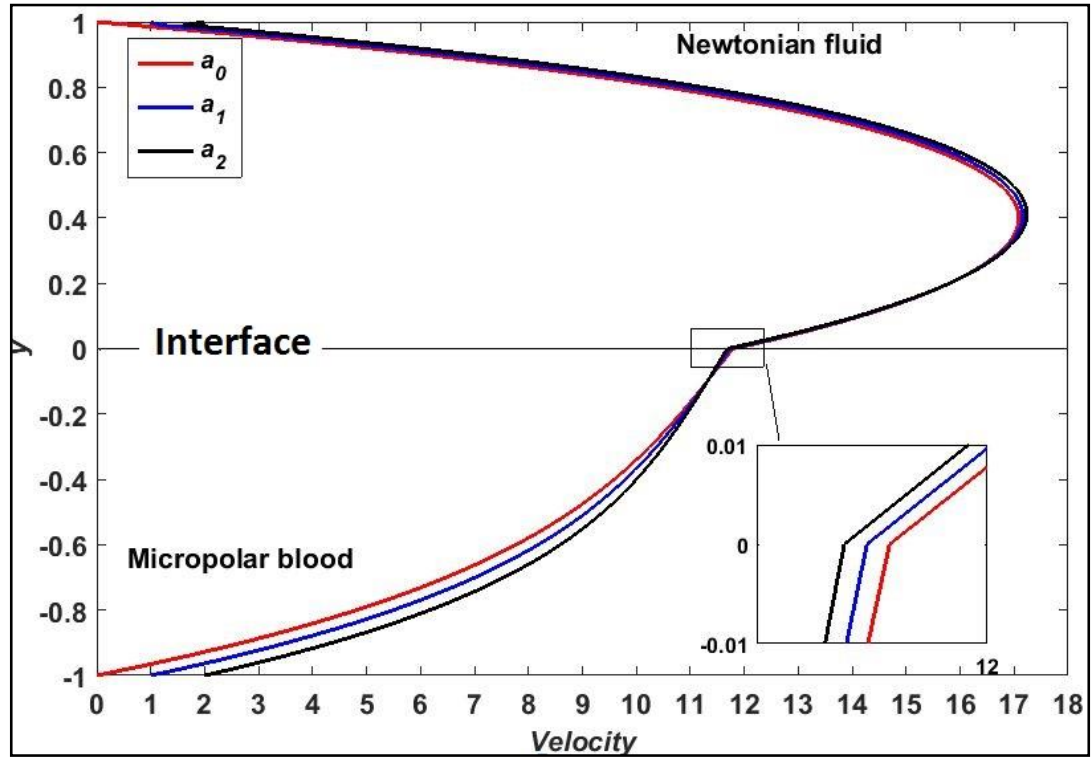

Figure 5. Velocity profile with the fuzzy boundary.

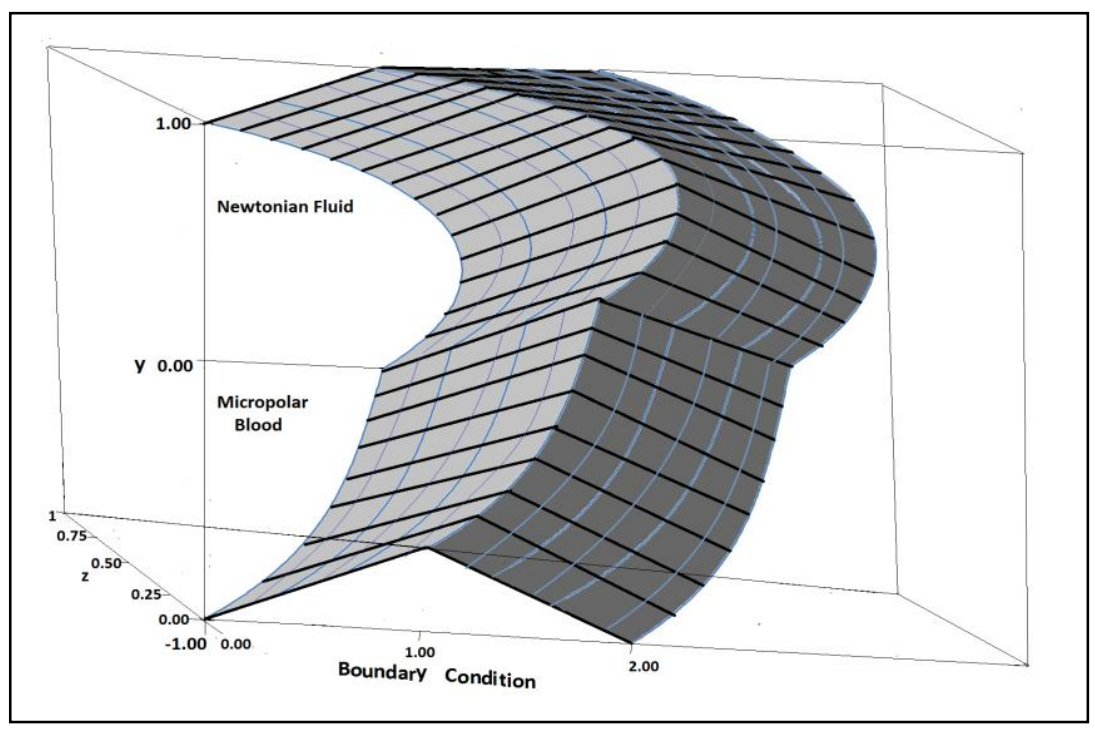

Figure 6. Evolution of membership grade for velocity profile with the fuzzy boundary. 
The above Figure 5 and Figure 6 shows the fuzzy solution of velocity profiles in the both region with the fuzzy boundary conditions $\left(\alpha_{c}, 1,2-\alpha_{c}\right)$ where $\alpha_{c} \rightarrow[0,1]$. As $\alpha_{c}$ is varying from 0 to 1 so the respective condition represents a triangular number $(0 ; 1 ; 2)$. In Figure 5 , the profiles $a_{0}$, $a_{1}$, and $a_{2}$ are the corresponding $\alpha$-cut solution to the endpoints $(0 ; 1 ; 2)$ of the boundary value and it is observed that the curvature of both fluid velocities is parabolic and initially, the velocity profiles of micropolar blood and Newtonian fluid increases with boundary value. As the lower region fluid is denser and more viscous than the upper region so the magnitude of the flow velocity of micropolar blood increases faster than Newtonian fluid and near the interface, both profiles show the decreasing nature.

Figure 6 exhibits the evolution of membership grade for the fuzzy solution of velocity profiles which with boundary $\left(\alpha_{c}, 1,2-\alpha_{c}\right)$. The degree of membership grade evolved from 0 to 1 as the boundary conditions at both the plates rising in the interval $[0,1]$ and membership profile declines with grades 1 to 0 as the boundary conditions arise in the interval $[1,2]$.

Bellow Figure 7 depicts the $\alpha_{c}$-levels of the fuzzy solution for velocity profile in the grayscale image. The scale ranging from white to black color corresponding respectively to $\alpha_{c}$ varying from 0 to 1 which shows that near the center of the triangular fuzzy boundary, the membership grade of the solution is high and near the lower vertex of the triangular fuzzy boundary, the membership grade of the solution is low.

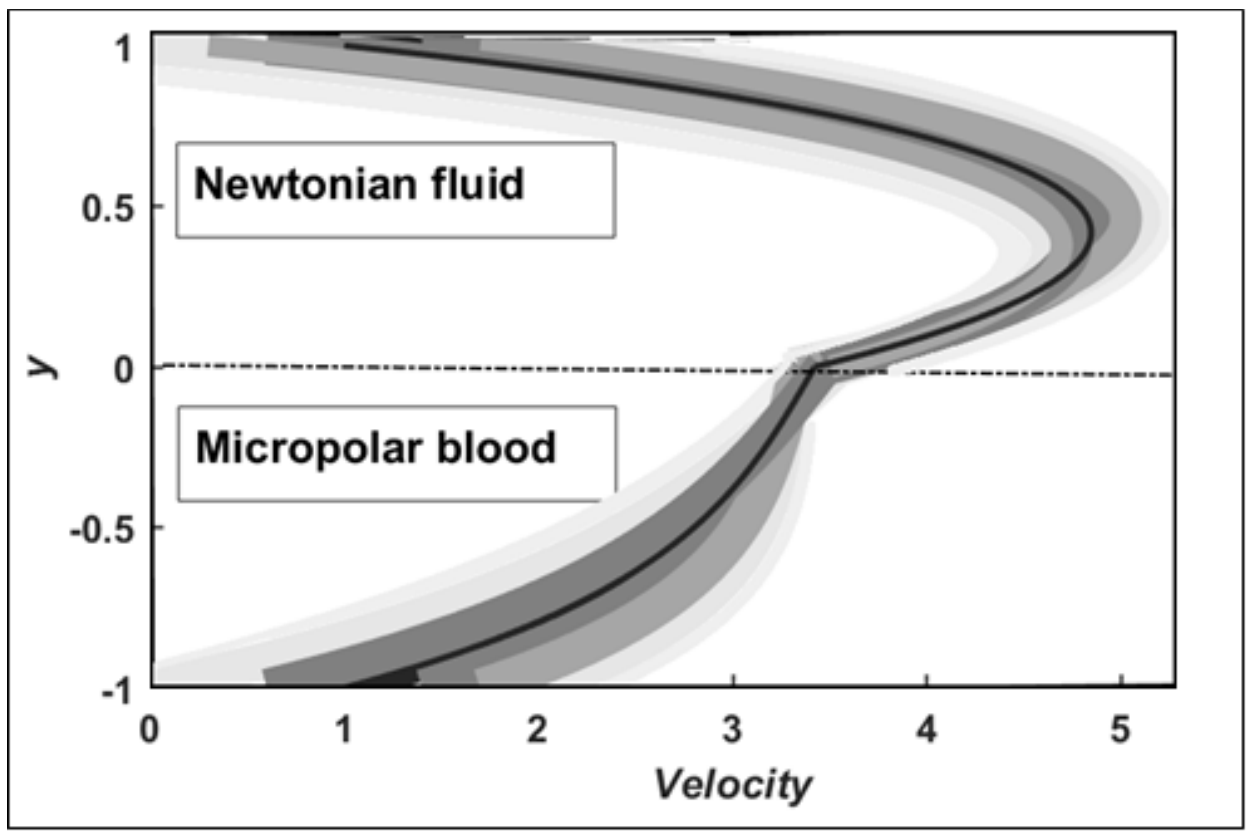

Figure 7. Grayscale image of fuzzy solution for velocity profile with the fuzzy boundary. 


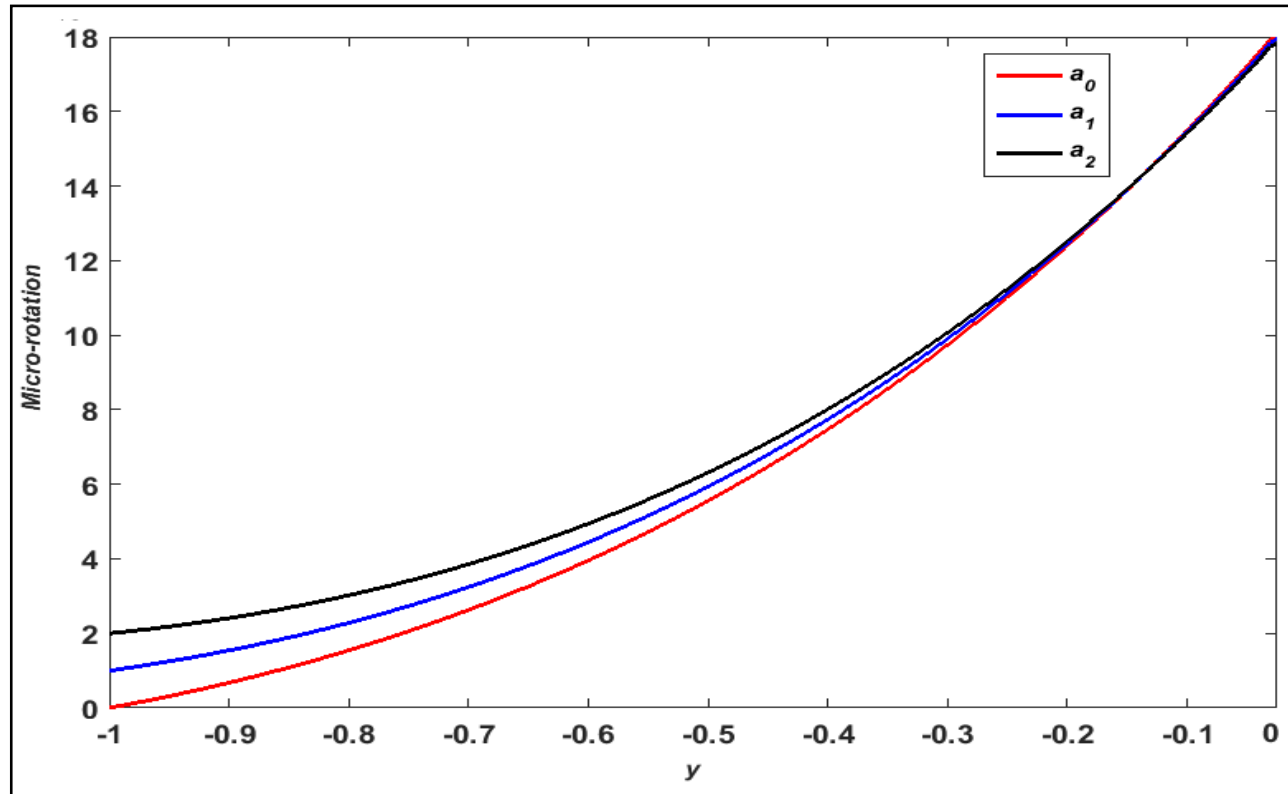

Figure 8. Micro-rotation profile with the fuzzy boundary.

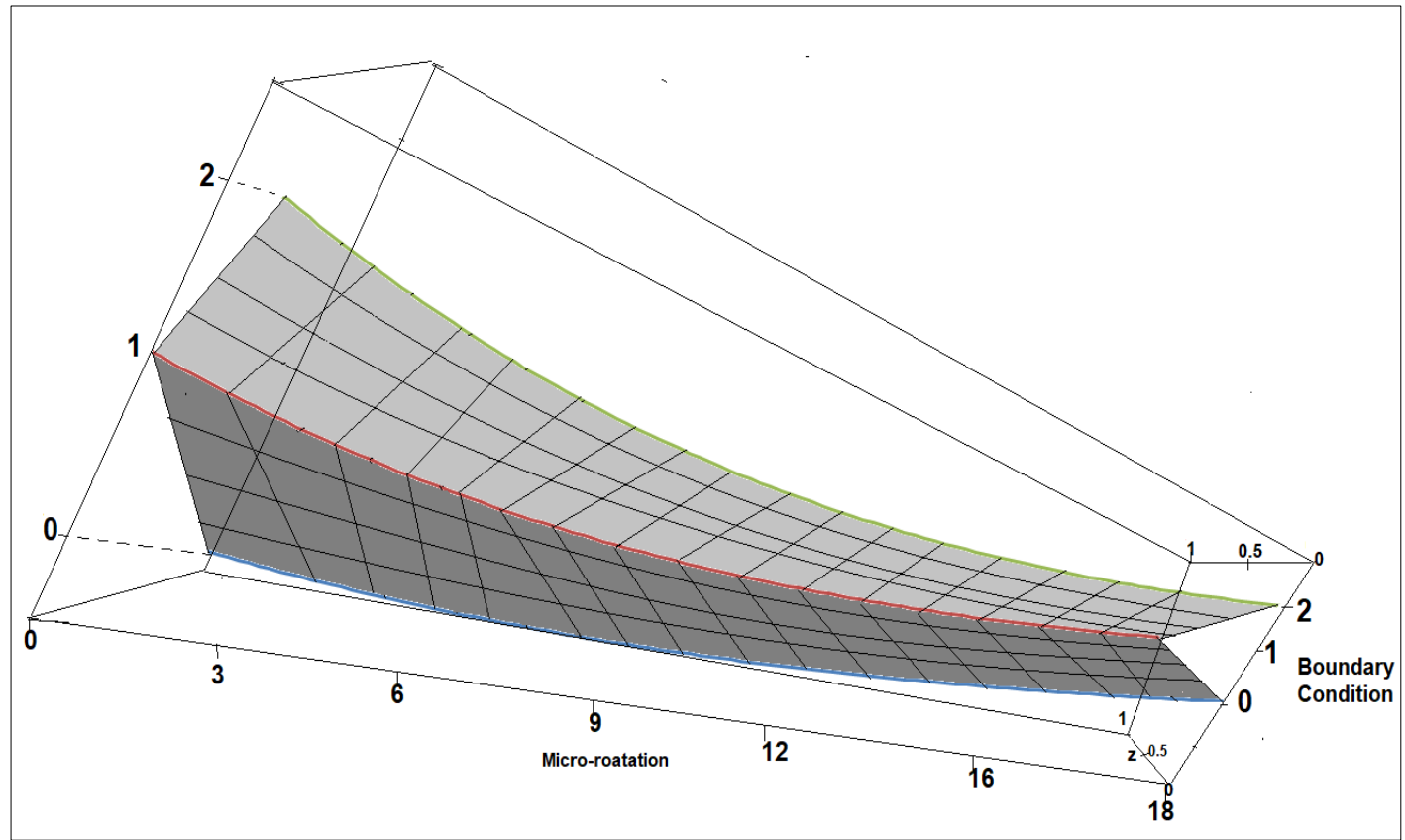

Figure 9. Evolution of membership grade for a micro-rotation profile with the fuzzy boundary.

The above Figure 8 and Figure 9 shows the fuzzy solution of micro-rotation profiles with fuzzy boundary condition $\left(\alpha_{c}, 1,2-\alpha_{c}\right)$ in the micropolar blood region where $\alpha_{c} \rightarrow[0,1]$. In Figure 8 the solution $a_{0}, a_{1}$, and $a_{2}$ corresponds to the endpoints $(0 ; 1 ; 2)$ of the $\alpha$-cut of the solution and it is 
observed that initially the micro-rotation vector profile of micropolar blood increases with boundary value and near the interface, it shows the decreasing nature. Figure 9 shows the fuzzy solution of micro-rotation profiles which exhibits the evolution of membership grade with boundary $\left(\alpha_{c}, 1,2-\alpha_{c}\right)$.

\section{Conclusions}

The fuzzy solution for the time-dependent flow of two immiscible micropolar blood and Newtonian fluids through a horizontal channel under fuzzy boundary conditions has been analyzed. An updated cubic B-spline differential quadrature technique for flow velocity and microrotation is presented. The calculated numerical results are validated under the limiting case of non-micropolar Newtonian fluid flow by comparing with the exact solution. The membership grade of fuzzy solutions for velocity and microrotation profiles has been depicted numerically and the $\alpha_{c}$-levels of the fuzzy solution for velocity and micro rotation profile are mentioned in the grayscale. The core outcomes of the current work are presented hereunder.

A strong correlation is seen between the numerical and exact solutions in the limiting situation as the absolute and root mean square errors are both so very small and also decreasing with time.

The velocity and micro-rotation vector profiles initially rise with fuzzy boundaries; however, the profiles exhibit a decreasing character towards the interface due to the micropolar parameter impact.

Due to boundary conditions, the change in micropolar fluid velocity is greater than the change in Newtonian fluid velocity. The velocity and microrotation patterns shift from increasing nature to decreasing near the interface.

The current work is relevant to biomedical and materials processing systems that can be extended with Trapezoidal fuzzy boundary conditions.

\section{Conflict of Interest}

The authors confirm that there is no conflict of interest to declare for this publication.

\section{Acknowledgments}

This research did not receive any specific grant from funding agencies in the public, commercial, or not-for-profit sectors. The authors would like to thank the editor and anonymous reviewers for their comments that help improve the quality of this work.

\section{References}

Abdel-Wahed, M.S. (2017). Lorentz force effect on mixed convection micropolar flow in a vertical conduit. The European Physical Journal Plus, 132(5), 1-11. https://doi.org/10.1140/epjp/i2017-11449-1.

Akbar, N.S., Tripathi, D., Khan, Z.H., \& Bég, O.A. (2018). Mathematical modelling of pressure-driven micropolar biological flow due to metachronal wave propulsion of beating cilia. Mathematical Biosciences, 301, 121-128. https://doi.org/10.1016/j.mbs.2018.04.001.

Ali, L., Liu, X., Ali, B., Mujeed, S., \& Abdal, S. (2019). Finite element analysis of thermo-diffusion and multi-slip effects on mhd unsteady flow of casson nano-fluid over a shrinking/stretching sheet with radiation and heat source. Applied Sciences, 9(23), 5217. https://doi.org/10.3390/app9235217. 
Apparao, S., Naduvinamani, N.B., \& Patil, M.D. (2013). Lubrication characteristics of porous inclined stepped composite bearings with couple stress fluids. Tribology Online, 8(3), 234-241.

Arora, G., \& Bhatia, G.S. (2020). Radial basis function pseudospectral method for solving standard fitzhughnagumo equation. International Journal of Mathematical, Engineering and Management Sciences, 5(6), 1488-1497. https://doi.org/10.33889/IJMEMS.2020.5.6.110.

Arora, G., Joshi, V., \& Mittal, R.C. (2019). Numerical simulation of nonlinear Schrödinger equation in one and two dimensions. Mathematical Models and Computer Simulations, 11(4), 634-648.

Barhoi, A., Hazarika, G.C., \& Dutta, P. (2018). Numerical study of fuzzified boundary value problem for couette type flow of fluid mechanics. Annals of Pure Applied Mathematics, 16(2), 373-384.

Bellman, R., Kashef, B.G., \& Casti, J. (1972). Differential quadrature: a technique for the rapid solution of nonlinear partial differential equations. Journal of Computational Physics, 10(1), 40-52.

Bird, R.B. (2002). Transport phenomena. Applied Mechanics. Reviews, 55(1), R1-R4. https://doi.org/10.1115/1.1424298.

Borah, J., Hazarika, G.C., \& Dutta, P. (2020). Numerical analysis of an unsteady mhd free convective fluid flow with uncertain parameters. Advances in Mathematics: Scientific Journal, 9(11), 9565-9574.

Borrelli, A., Giantesio, G., \& Patria, M.C. (2017). Reverse flow in magnetoconvection of two immiscible fluids in a vertical channel. Journal of Fluids Engineering, 139(10), 101203.

Chamkha, A.J., Yassen, R., Ismael, M.A., Rashad, A.M., Salah, T., \& Nabwey, H.A. (2020). MHD free convection of localized heat source/sink in hybrid nanofluid-filled square cavity. Journal of Nanofluids, 9(1), 1-12. https://doi.org/10.1166/jon.2020.1726.

Chaube, M.K., Pandey, S.K., \& Tripathi, D. (2010). Slip effect on peristaltic transport of micropolar fluid. Applied Mathematics Sciences, 4(43), 2015-2117.

Chen, Y.Y., Hsiao, R.J., \& Huang, M.C. (2018). Fuzzy solutions for two-dimensional Navier-Stokes equations. Journal of Mechanics, 34(1), 1-10. https://doi.org/10.1017/jmech.2015.92.

Chetia, B.C., \& Hazarika, G.C. (2000). Effects of fuzziness on dynamical similarity and Reynold's number. Fuzzy Sets and Systems, 115(3), 463-469. https://doi.org/10.1016/S0165-0114(98)00361-3.

Civan, F. (1993). Comment on 'application of generalized quadrature to solve two-dimensional incompressible Navier Stokes equations', By C. Shu and B. E Richards. International Journal for Numerical Methods in Fluids, 17(10), 921-922. https://doi.org/10.1002/fld.1650171007.

Devakar, M., \& Iyengar, T.K.V. (2013). Unsteady flows of a micropolar fluid between parallel plates using state space approach. The European Physical Journal Plus, 128(4), 1-13.

Devakar, M., \& Raje, A. (2018). A study on the unsteady flow of two immiscible micropolar and Newtonian fluids through a horizontal channel: a numerical approach. The European Physical Journal Plus, 133(5), 1-14. https://doi.org/10.1140/epjp/i2018-12011-5.

Eringen, A.C. (1968). Mechanics of micromorphic continua. In Mechanics of generalized continua. IUTAM Symposia (International Union of Theoretical and Applied Mechanics). Springer, Berlin, Heidelberg. https://doi.org/10.1007/978-3-662-30257-6_2.

Farooq, A.A., Tripathi, D., \& Elnaqeeb, T. (2019). On the propulsion of micropolar fluid inside a channel due to ciliary induced metachronal wave. Applied Mathematics and Computation, 347, 225-235.

Hamid, M., Zubair, T., Usman, M., \& Haq, R.U. (2019). Numerical investigation of fractional-order unsteady natural convective radiating flow of nanofluid in a vertical channel. AIMS Mathematics, 4(5), 1416-1429. 
Jiang, C., Zhang, Z.Q., Han, X., Liu, G., \& Lin, T. (2018). A cell-based smoothed finite element method with semi-implicit CBS procedures for incompressible laminar viscous flows. International Journal for Numerical Methods in Fluids, 86(1), 20-45.

Jiwari, R., \& Alshomrani, A.S. (2017). A new algorithm based on modified trigonometric cubic B-splines functions for nonlinear Burgers'-type equations. International Journal of Numerical Methods for Heat \& Fluid Flow, 27(8), 1638-1661.

Kang, C.K., \& Eringen, A.C. (1976). The effect of microstructure on the rheological properties of blood. Bulletin of Mathematical Biology, 38(2), 135-159.

Katta, R., Chandrawat, R.K., \& Joshi, V. (2020b, May). A numerical study of the unsteady flow of two immiscible micro polar and Newtonian fluids through a horizontal channel using DQM with B-spline basis function. In Journal of Physics: Conference Series, (Vol. 1531, No. 1, p. 012090). IOP Publishing.

Katta, R., Tripathi, D., Bhatti, M.M., \& Khalique, C.M. (2020a). Electro-osmotic flow of hydromagnetic dusty viscoelastic fluids in a microchannel propagated by peristalsis. Journal of Molecular Liquids, 314, 113568.

Mekheimer, K.S., \& El Kot, M.A. (2008). The micropolar fluid model for blood flow through a tapered artery with a stenosis. Acta Mechanica Sinica, 24(6), 637-644.

Mittal, R.C., \& Jain, R.K. (2012). Numerical solutions of nonlinear Burgers' equation with modified cubic B-splines collocation method. Applied Mathematics and Computation, 218(15), 7839-7855.

Peddieson Jr, J. (1972). An application of the micropolar fluid model to the calculation of a turbulent shear flow. International Journal of Engineering Science, 10(1), 23-32.

Quan, J.R., \& Chang, C.T. (1989a). New insights in solving distributed system equations by the quadrature method-I. analysis. Computers \& Chemical Engineering, 13(7), 779-788.

Quan, J.R., \& Chang, C.T. (1989b). New insights in solving distributed system equations by the quadrature method-II. numerical experiments. Computers \& Chemical Engineering, 13(9), 1017-1024.

Rajeev, S.G. (2008). Fuzzy fluid mechanics in three dimensions. International Journal of Modern Physics A, 23(07), 1019-1028. https://doi.org/10.1142/S0217751X08038615.

Ramesh, K., \& Joshi, V. (2019). Numerical solutions for unsteady flows of a magnetohydrodynamic jeffrey fluid between parallel plates through a porous medium. International Journal for Computational Methods in Engineering Science and Mechanics, 20(1), 1-13.

Shan, Y.Y., Shu, C., \& Lu, Z.L. (2008). Application of local MQ-DQ method to solve 3D incompressible viscous flows with curved boundary. Computer Modeling in Engineering and Sciences, 25(2), 99-13.

Shu, C., \& Richards, B.E. (1992). Application of generalized differential quadrature to solve two-dimensional incompressible Naiver-Stocks equations. International Journal of Numerical Methods in Fluids, 15(7), 791-798.

Shu, C., Shan, Y.Y., \& Qin, N. (2007). Development of a local MQ-DQ-based stencil adaptive method and its application to solve incompressible Navier-Stokes equations. International Journal for Numerical Methods in Fluids, 55(4), 367-386.

Shuaib, M., Ali, A., Khan, M.A., \& Ali, A. (2020). Numerical investigation of an unsteady nanofluid flow with magnetic and suction effects to the moving upper plate. Advances in Mechanical Engineering, 12(2), 1-13.

Singh, A.K. (2005). Convective flow of two immiscible viscous fluids using Brinkman model. Indian Journal of Pure and Applied Physics, 43(6), 415-421

Singh, A.K., Singh, A.K., \& Singh, N.P. (2004). Generalised couette flow of two immiscible viscous fluids with heat transfer using Brinkman model. Heat and Mass Transfer, 40(6), 517-523. 
Srinivas, J., \& Murthy, J.V.R. (2016a). Thermal analysis of a flow of immiscible couple stress fluids in a channel. Journal of Applied Mechanics and Technical Physics, 57(6), 997-1005.

Srinivas, J., \& Murthy, J.V.R. (2016b). Flow of two immiscible couple stress fluids between two permeable beds. Journal of Applied Fluid Mechanics, 9(1), 501-507.

Srinivas, J., Murthy, J.V.R., \& Sai, K.S. (2015). Entropy generation analysis of the flow of two immiscible couple stress fluids between two porous beds. Computational Thermal Sciences: An International Journal, 7(2), 123-137.

Tripathi, D., Prakash, J., Reddy, M.G., \& Misra, J.C. (2021). Numerical simulation of double diffusive convection and electroosmosis during peristaltic transport of a micropolar nanofluid on an asymmetric microchannel. Journal of Thermal Analysis and Calorimetry, 143(3), 2499-2514.

Umavathi, J.C., Chamkha, A.J., Manjula, M.H., \& Al-Mudhaf, A. (2005). Flow and heat transfer of a couplestress fluid sandwiched between viscous fluid layers. Canadian Journal of Physics, 83(7), 705-720.

Umavathi, J.C., Kumar, J.P., \& Chamkha, A.J. (2008). Flow and heat transfer of a micropolar fluid sandwiched between viscous fluid layers. Canadian Journal of Physics, 86(8), 961-973.

Umavathi, J.C., Kumar, J.P., \& Chamkha, A.J. (2009). Convective flow of two immiscible viscous and couple stress permeable fluids through a vertical channel. Turkish Journal of Engineering and Environmental Sciences, 33(4), 221-243.

Vaid, M.K., \& Arora, G. (2019). Quintic B-spline technique for numerical treatment of third order singular perturbed delay differential equation. International Journal of Mathematical, Engineering and Management Sciences, 4(6), 1471-1482.

Wang, L., Jian, Y., \& Li, F. (2016). The flow of micropolar fluids through a microparallel corrugated channel. The European Physical Journal Plus, 131(9), 1-12.

Ziabakhsh, Z., Domairry, G., \& Bararnia, H. (2009). Analytical solution of non-Newtonian micropolar fluid flow with uniform suction/blowing and heat generation. Journal of the Taiwan Institute of Chemical Engineers, 40(4), 443-451. 